\title{
Preparation of bismuth-based microrods and their application in electroanalysis
}

\author{
Xueping $\mathrm{Hu}^{\mathrm{a}, \mathrm{b}}$, Dawei Pan ${ }^{\mathrm{a}, \mathrm{b}, *}$, Haitao Han ${ }^{\mathrm{a}}$, Mingyue Lin ${ }^{\mathrm{a}, \mathrm{b}}$, Yun $\mathrm{Zhu}^{\mathrm{a}, \mathrm{b}}$, Chenchen Wang ${ }^{\mathrm{a}}$ \\ ${ }^{a}$ Key Laboratory of Coastal Environmental Processes and Ecological Remediation, Yantai Institute of Coastal Zone Research (YIC), Chinese Academy of Sciences (CAS), \\ Shandong Provincial Key Laboratory of Coastal Environmental Processes, YICCAS, Yantai, Shandong 264003, PR China \\ ${ }^{\mathrm{b}}$ University of Chinese Academy of Sciences, Beijing 100049, PR China
}

\section{A R T I C L E I N F O}

\section{Article history:}

Received 30 August 2016

Received in revised form 28 December 2016

Accepted 30 December 2016

Available online 31 December 2016

\section{Keywords:}

Bismuth

Microstructure

XPS

Electroanalysis

Iron

\begin{abstract}
A B S T R A C T
Bismuth-based microrods (BiMRs) were synthesized through a single reductant-controlled chemical reduction method. With the various quantity of reductant, different morphologies of Bi based materials were obtained. Characters of BiMRs were investigated by using scanning electron microscope (SEM), Xray diffraction (XRD) and X-ray photoelectron spectroscopy (XPS). The results showed that the product was uniform rods with a smooth surface and neat edges. Furthermore, BiMRs was consisted of bismuth and bismuth oxide which made the material possess both advantages of metal and metal oxide. BiMRs had shown excellent electrochemical performance and was successfully used for the determination of total dissolved iron in real water samples. Considering their environmentally friendly and excellent electrochemical performance, BiMRs might be used as a promising electrode material in electroanalysis field. (c) 2016 Elsevier B.V. All rights reserved.
\end{abstract}

\section{Introduction}

Electroanalysis has paid great attention for the low cost and high sensitivity. But for many years, electroanalytical techniques were associated with the use of mercury electrodes for the trace elements detection. This liquid metal had dominated in electrochemistry for lengthy decades [1]. But now, efforts are mainly directed towards development of 'environmentally friendly' solid electrodes, functionalized micro/nano-structured interfaces modified electrodes [2].

Bismuth-based material as green material has been intensively studied in the couple of decades due to their unique properties, which enables them to be promising in very broad areas including catalysis, photonics, electronics, optoelectronics, energy storage, sensor, superconductive material and electroanalysis [3]. Bismuth which knew as an environmentally friendly material has received significant attention as an alternative to commonly used toxic mercury in electroanalysis. A wide variety of bismuth-based electrodes, sensors, and detectors of various types, configurations, or constructions had been reported by many researchers [4]. Among

\footnotetext{
* Corresponding author at: Key Laboratory of Coastal Environmental Processes and Ecological Remediation, Yantai Institute of Coastal Zone Research (YIC), Chinese Academy of Sciences (CAS), Shandong Provincial Key Laboratory of Coastal Environmental Processes, YICCAS, Yantai, Shandong 264003, PR China.

E-mail address: dwpan@yic.ac.cn (D. Pan).
}

them, bismuth film electrodes were mostly used (BiFE) [5,6]. Metallic modifiers ( $\mathrm{Bi}$ ) would have a better improvement effect when the underlying electrode substrates such as boron doped diamond electrode (BDDE) and glassy carbon electrode (GCE) exhibit relatively slow electron transfer properties [7], but it was not very stable and easy to be oxidized [8,9]. Especially when underlying electrode substrate exhibits fast electron transfers kinetics, the improvements of using BiFE modified electrode were not apparent and in some cases can lead to a detrimental effect upon the electroanalytical response [7]. Then other forms of bismuth, such as bismuth nanoparticles, bismuth nanosheets were reported as modified materials in electroanalysis $[10,11]$, and the change of the morphology improved those materials' stabilities to some extent. Besides, bismuth oxide modified carbon electrode [12] and screen printed electrode [13] had also been employed for the determination of heavy metal ions due to its characteristic parameters such as the energy band gap, and photoconductivity.

In this paper, bismuth-based microrods (BiMRs) which contained both metal bismuth and bismuth oxide were synthesized through chemically hydrothermal reduction method by using sodium borohydride as a reducing agent. Bismuth-based materials with different components and morphologies were obtained by controlling the ratio of reactant and reductant. BiMRs had both the merits of metal bismuth and bismuth oxide and could be used directly without any modified treatment. Bismuth-based microrods material modified glassy carbon electrode (BiMRs/GEC) had a wider working potential window than just BiFE and bismuth 
oxide modified electrode. Besides, this bismuth-based microrods material was successfully applied to the determination of iron in real coastal waters and had great potential in electroanalysis.

\section{Experimental}

\subsection{Preparation of bismuth-based material}

Related chemicals and reagents were shown in Supporting information S1. Bismuth-based materials were synthesized by chemically reduction method. Firstly, bismuth nitrate solution was prepared by the primary dissolving bismuth nitrate with distilled water and then transferred to a round-bottom flask. After putting the flak into an oil bath pan at the temperature of $30^{\circ} \mathrm{C}$ with stirring, a freshly prepared sodium borohydride solution was dropped into the flak by using constant pressure funnel. With the addition of sodium borohydride, insoluble substances appeared. Finally, the product was filtered and washed several times with distilled water exhaustively and then dried under vacuum at $60^{\circ} \mathrm{C}$ for $4 \mathrm{~h}$. Different morphologies and components of bismuth-based materials were obtained through controlling the ratio of bismuth nitrate and reductive agent. BiMRs would be synthesized at the ratio of 6 .

\subsection{Characterization}

Surface morphology was observed by scanning electron microscopy (SEM Hitachi S-4800 microscope). To analyze the crystal structures of the samples, the X-ray diffraction (XRD) measurements were conducted on an X-ray diffractometer (XRD-7000, Shimadzu Corporation) from $10^{\circ}$ to $80^{\circ}$. Surface chemistry was carried out using X-ray photoelectron spectroscopy (XPS Kratos Amicus) with $\mathrm{Mg} \mathrm{K \alpha}$ irradiation.

\subsection{Electrochemical detection of iron}

The detection of iron was investigated by differential pulse voltammetry (DPV) on electrochemical workstation (CHI 660D, Chenhua Instruments, Shanghai, China) using a three electrode system. BiMRs/GCE was used as the working electrode and the dosage of modified materials had been optimized. Preparation of the modified electrode and determination of real samples had shown in Supporting information S2.

\section{Results and discussion}

\subsection{Surface morphology}

Fig. 1 showed the SEM images of bismuth-based materials with different ratios of bismuth nitrate and reducing agent. When the ratio $(\mathrm{n} / \mathrm{n})$ was 6 , the inerratic microrods can be seen under different magnification (Fig. $1 \mathrm{a}$ and $\mathrm{b}$ ). The microrods were uniformly distributed with an average length of $10 \mu \mathrm{m}$ and width of $2 \mu \mathrm{m}$. All those rods were angular cuboid with precisely ridge and a smooth surface. When the ratio of bismuth nitrate and reducing agent reduced to 4.5 , the morphology of bismuth-based material was shown in Fig. S1a. With the relatively decreasing of bismuth nitrate, a number of porous bismuth appeared instead of microrods. The bismuth-based porous material had no regular morphological form and was composed by cloddy substance with a number of holes. A lot of nanoparticles with diameter of 7$10 \mathrm{~nm}$ were seen in Fig. S1b when the ratio was 3. From the SEM images in Fig. 1 and Fig. S1, it can be concluded that the volume of bismuth-based materials either decreased and the shape also had a big variation with the decreasing ratio of bismuth nitrate and reducing agent. It was speculated that when the ratio was bigger, bismuth nitrate was relatively excess comparing to reducing agent. In this case, bismuth nitrate cannot be reduced to bismuth completely instead of some bismuth oxide. Then bismuth oxide would assemble to become a big mass. Meanwhile, the existence of bismuth oxide might affect the crystalline growth direction and result in the formation of microrods. In contrast, bismuth nitrate would be reduced to metal bismuth mostly when the reducing agent was relatively surplus than reducing agent. The products were small porous or nanoparticles substances. Besides the ratio of reagents, reaction temperature was also investigated and the results showed that the reaction temperature had no substantial influence on the materials' morphology.

\subsection{Component of synthesized materials}

XRD patterns of the bismuth-based nanoparticles and BiMRs were shown in Fig. S2a and b. Typical diffraction peaks in Fig. S2a at $2 \theta=27.19^{\circ}, 37.88^{\circ}$ and $39.61^{\circ}$ corresponding to the (012), (104), (110) pure rhombohedral metal bismuth phase (JCPDS card, PDF No. 44-1246) with no characteristic peaks from other impurities. But the spectrum in Fig. S2b was very complicated. Comparing with the PDF card, in addition to the peaks of metal bismuth, there were many peaks belonging to bismuth oxide. So, it was concluded that BiMRs contained not only metal bismuth but also a mass of bismuth oxide. Fig. $2 \mathrm{a}$ and $\mathrm{b}$ were the narrow XPS spectra of O1s (a) and Bi4f (b) on BiMRs. The 01s peak of BiMRs in Fig. 2a at a binding energy of about $530 \mathrm{eV}$. Two asymmetrical main peaks observed at 159.42 and $164.76 \mathrm{eV}$ in Fig. $2 \mathrm{~b}$ correspond to the $\mathrm{Bi} 4 \mathrm{f} 7 / 2$ and $4 \mathrm{f} 5 / 2$ signals, respectively [10]. These peaks implied that the bismuth was present in the $\mathrm{Bi}^{3+}$ oxidation state, consistent with previous findings. Two other small peaks can also be noticed in the spectrum in Fig. 2b, corresponding to binding energies of 158.86 and $163.67 \mathrm{eV}$. These peaks were
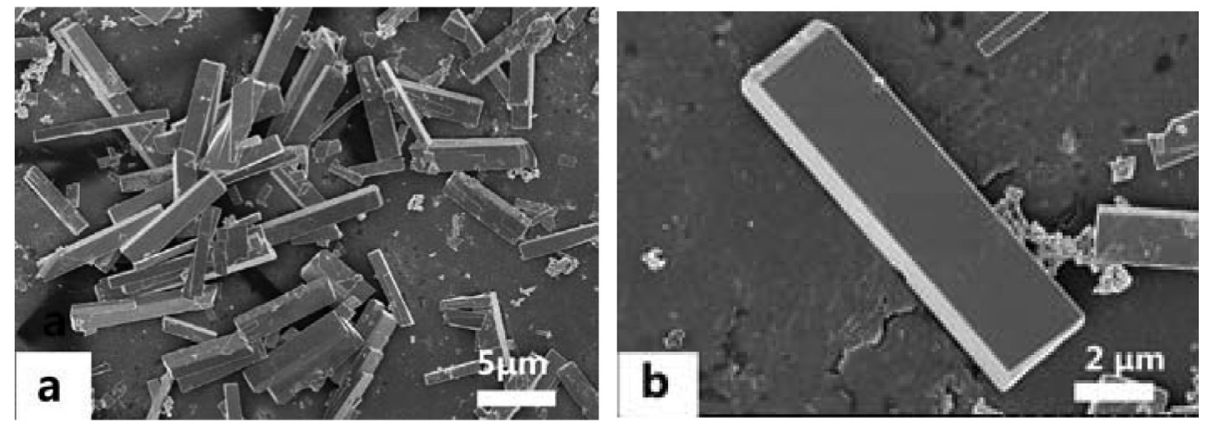

Fig. 1. SEM image of bismuth-based microrods (a and b). 

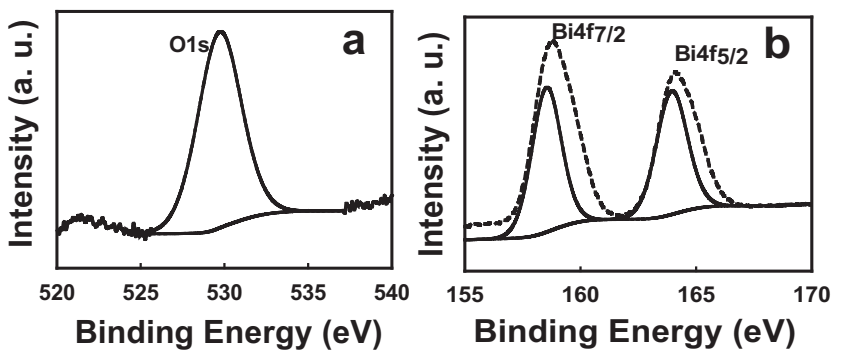

Fig. 2. XPS narrow spectra of O1s (a) and Bi 4f (b) on BiMR.
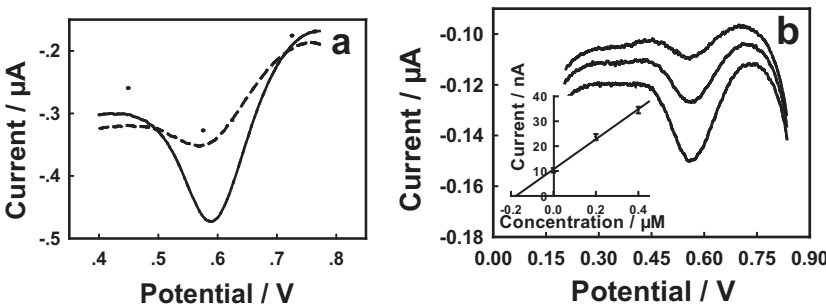

Fig. 3. DPVs of BiMRs/GCE (solid line), porous bismuth material modified GCE (dotted line) and bismuth nanoparticles modified GCE (dashed line) in $0.05 \mathrm{M} \mathrm{HCl}$ solution containing $1 \mu \mathrm{M} \mathrm{Fe}(\mathrm{III})$ (a) and typical voltammograms for the determination of $\mathrm{Fe}(\mathrm{III})$ in coastal river sample (b).

agreed with the Bi $4 \mathrm{f}$ signals for Bi metal [14], indicating the BiMRs product surely contains the metal bismuth and bismuth oxide.

\subsection{Electrochemical property and application of microrodes}

To testify electrochemical property of various Bi-based materials, three modified electrode were investigated for the determination of $1 \mu \mathrm{M} \mathrm{Fe}(\mathrm{III})$ and results had showed in Fig. 3a. It can be observed that the peak current obtained at BiMRs/GCE was higher than the porous bismuth material modified GCE and bismuth nanoparticles modified GCE. It was supposed that the existence of bismuth oxide might overcome the problems that bismuth film electrode was instability in positive potential and easy to hydrolyze. BiMRs/GEC had a wider working potential window which can detect the reduction of $\mathrm{Fe}(\mathrm{III})$ in positive working potential continuously and directly. Additionally, the effect of different $\mathrm{pH}$ values of electrolyte solution on the electrochemical response of $\mathrm{Fe}(\mathrm{III})$ were examined and the corresponding results had shown in Fig. S3. The largest response was obtained at $\mathrm{pH}$ $1.3(0.05 \mathrm{M} \mathrm{HCl})$. Besides, the calibration curve of the $\mathrm{Fe}(\mathrm{III})$ determination was derived from the DPVs obtained at the BiMRs/GEC. As shown in Fig. S4, the resulting calibration plots had a favorable linear in the range of $0.02-10 \mu \mathrm{M}$. The detection limit of $\mathrm{Fe}(\mathrm{III})$ at a BiMRs/GEC was $6.4 \mathrm{nM}(\mathrm{s} / \mathrm{n}=3)$. Furthermore, BiMRs/GCE was firstly used for the total dissolved iron determination in coastal water samples by using the standard addition method.
The adsorptive cathodic stripping curves of the real water sample were shown in Fig. $3 \mathrm{~b}$. Peak currents were linear with the concentrations (the inset in Fig. $3 b$ ) and the equation was ip $=60.54 \mathrm{C}-10.72$ with a correlation coefficient of 0.998 . The concentration of total dissolved iron was calculated to be $177.07 \mathrm{nM}$ and the result was in agreement with the value detected by atomic absorption spectroscopy, indicating the capability and accuracy of the BiMRs/GCE for iron determination in real water samples.

\section{Conclusion}

BiMRs material was synthesized through controlling the condition of chemical reduction reaction. The obtained products were exhibited as uniform rods with neat edges by SEM characterization. This material was proved not only contained mental bismuth but also contained oxide bismuth through XPS and XRD characterizations. So, the BiMRs had both the merits of metal bismuth and bismuth oxide. Finally, this novel microrods material was successfully used for the determination of total dissolved iron in real water samples with good accuracy and precision.

\section{Acknowledgements}

This work was financially supported by the National Natural Science Foundation of China (41276093), the Youth Innovation Promotion Association (2011170), the Outstanding Young Scientists Program and the Equipment Development Project of Chinese Academy of Sciences (YZ201558).

\section{Appendix A. Supplementary data}

Supplementary data associated with this article can be found, in the online version, at http://dx.doi.org/10.1016/j.matlet.2016.12. 119.

\section{References}

[1] G. Munteanu, S. Munteanu, D.O. Wipf, J. Electroanal. Chem. 632 (2009) 177183.

[2] D. Omanović, C. Garnier, K.G. Walsh, I. Pižeta, Electrochem. Commun. 61 (2015) 78-83.

[3] A. Scarangella, R. Reitano, G. Franzò, F. Priolo, M. Miritello, Appl. Phys. Lett. 107 (2015) 041908.

[4] J. Wang, Electroanalysis 17 (2005) 1341-1346.

[5] R. Segura, M. Toral, V. Arancibia, Talanta 75 (2008) 973-977.

[6] M. Chamjangali, H. Kouhestani, F. Masdarolomoor, H. Daneshinejad, Sens. Actuators, B 216 (2015) 384-393.

[7] C.W. Foster, A.P. Souza, J.P. Metters, M. Bertotti, C.E. Banks, Analyst 140 (2015) $7598-7612$.

[8] L. Pinto, S.G. Lemos, Electroanalysis 26 (2014) 299-305.

[9] L. Chen, Z.H. Su, X.H. He, Y. Liu, C. Qin, Y.P. Zhou, Z. Li, L.H. Wang, Q.J. Xie, S.Z. Yao, Electrochem. Commun. 15 (2012) 34-37.

[10] X. Hu, D. Pan, M. Lin, H. Han, F. Li, ECS Electrochem. Lett. 4 (2015) H43-H45.

[11] L.Z. Pei, T. Wei, N. Lin, H. Zhang, J. Alloys Compd. 663 (2016) 667-685.

[12] R. Pauliukaite, R. Metelka, I. Švancara, A. Królicka, A. Bobrowski, K. Vytřas, E. Norkus, K. Kalcher, Anal. Bioanal. Chem. 374 (2002) 1155-1162.

[13] R.O. Kadara, N. Jenkinson, C.E. Banks, Electroanalysis 21 (2009) 2410-2414.

[14] B. Sarma, A.L. Jurovitzki, Y.R. Smith, M. Misra, A.C.S. Appl, ACS Appl. Mater. Int. 5 (2013) 1688-1697. 